\title{
Considerations on radiotherapy dose intensity for limited small cell lung cancer
}

\author{
Andrew T. Turrisi \\ Department of Radiation Oncology, The Unicersity of Michigan Medical Center, Ann Arhor, \\ MI 48109, USA
}

\begin{abstract}
The factors of dose, volume, fractionation, and timing with chemotherapy undoubtedly influence outcomes in terms of treatment of limited small cell lung cancer with thoracic radiotherapy. The type and timing with chemotherapy may be very important. For integration of chemotherapy with radiation therapy, the measure of iso-effects, for tumor and acute tissue and late effects, may be hard to come by. This paper relates a variety of different total doses, according to the relative scales provided by nominal standard dose (NSD, NRET) and the biologic effective dose (Gy-10 and Gy-3), which employs the alphabeta linear quadratic model. A variety of different fraction schemes have been used clinically. These allow us to compare intensifying of the dose versus standard treatment versus relative prolongation of the dose. When defined as measure of 2-year survivals, there is not a tremendous difference in observed outcomes. However, there may be differences that are discerned later when the endpoint local control is examined. This paper reviews the current pilot studies using platinum-etoposide chemotherapy, at a variety of different dose-intensive regimens of thoracic radio-therapy and their relative effects. To prove benefit, randomized trials are needed.
\end{abstract}

Key words: Platinum-etoposide; Limited small cell lung cancer; Radiotherapy dose; Accelerated and hyperfractionated radiotherapy

\section{Introduction}

Although the progress that has been achieved overall has been slow over the last decade, there has been steady improvement in the median survivals and 2-year survivals for limited small cell lung cancer (SCLC) when compared to trials 
published in 1983 [2]. Then, the median survivals approximated 1-year, 2-year survivals were, at best, 14-17 months [3]. Now the medians approach 2 years, and local control may be $90 \%$ or better. There are a number of explanations for this including stage migration and selection factors, all of which influence patient entry onto trials. However, better chemotherapeutics with Platinum Etoposide-based programs (instead of cyclophosphamide and/or Adriamycin (doxorubicin)-based programs) and improved abilities to deliver radiotherapy in concurrent or alternating schemes may have contributed to these improved results.

A major reason to add radiotherapy to combined modality trials in small cell lung cancer is the approximately $80-90 \%$ local failure rate with chemotherapy alone. Even with the addition of radiotherapy in low doses, the failure rate still approximates $80 \%$ at lower doses and in higher doses it remains at roughly $30 \%$ [3]. The best local control rate published is $97 \%$ after $60 \mathrm{~Gy}$ was used with an infrequently used chemotherapy regimen [15]. The median and 2-year survival were too short, perhaps reflecting poor systemic control with this chemotherapy program.

Table 1 displays factors that may influence the relative merits of different schedules for administration of thoracic radiotherapy. Dose must be discussed both in terms of physical dose, expressed in rad or Gy, and relative biologic dose, expressed as RETs or Gy - 10s for acute effects, and 'neurets' or Gy - 3s for late effects. The relative biologic dose is influenced by the fractionation scheme, the time elapsed during treatment, the dose-per-fraction, the chemotherapy agents, as well as the factors listed in Table 1 . Some chemothcrapcutic agents may cause an increase in adverse effects. When certain strategies of thoracic radiotherapy are employed and with particular chemotherapeutics, such as Doxorubicin, bleomycin, mitomycin- $C$, or nitrosoureas, the net effect may be an increase in lung tissue toxicity. Successful combined modality trials produce increased efficacy against the cancer without causing excess toxicity to normal tissues. Leliveldt has nicely reported results of this using murine models for tumor and normal tissue endpoints [10].

The volume is another important factor that needs attention in lung cancer trials. Although volumes have increased in lung cancer therapy, no critical analysis

Table 1

Radiotherapy factors

Dose

Volume

Fractionation

Timing

Early vs. late

With chemotherapy

(a) Concurrent

(b) Alternating

(c) Sequential fashion 
shows that larger volumes improve survival or significantly enhance local control. Many have increased the volumes because of the anatomic knowledge of the lymphatics, or their frequency of involvement or potential for failure in those sites. Because of the therapeutic successes achieved in lymphomas, such as Hodgkin's disease, which are expanded field radiotherapies, the analogy dictates to lung cancer radiotherapy portals. Current models predicting late pulmonary effects implicate volume as a critical factor for lung [4,5]. Systems are now being tested to determine new levels for partial-organ tolerances. Prediction of complication may vary amongst different normal tissues that may have varied dependencies on volume, dose, and slope of curves describing specific events. For some tissues, like lung, volume is likely to be quite important, perhaps much more important than dose.

Timing of radiotherapy with chemotherapy may be quite important. The Looney hypothesis integrated radiotherapy with chemotherapy in novel ways [12]. In the laboratory, this resulted in enhanced cure rates. When brought to the clinic, reduction of toxicity proved another important goal. Alternating weeks of chemotherapy with weeks of radiotherapy allows relatively full doses of both modalities. However, this commonly requires split-course radiotherapy, which has fallen from favour because it allows tumor repopulation between courses. This strategy has been attempted in small cell lung cancer; however, with the chemotherapeutics used and in the schedules employed, despite increasing the dose from 45 to 55 , and then $65 \mathrm{~Gy}, 3$-year actuarial figures continued to show $70 \%$ control, no improvement in terms of local control [1]. Survival was adequate and comparable to other studies of the era, but the French studies that attempted alternating therapy failed to provide the desired goal of better local therapy with lesser toxicity [1].

One reason for local failure may be technical, i.e. inadequate or suboptimal coverage of the tumor bed. The spinal cord has been the focus of concern in the past. Many thoracic radiotherapy techniques have used posterior spinal cord blocks. With the use of simulation and CT-directed treatment planning systems, inclusion of target volumes and exclusion of critical normal tissues can be assured. Future studies will focus on normal tissue complication. Today computerized scans aid in diagnosis and therapy planning facilitate radiation of the target, but also assure protection of critical tissues.

The concept of isoeffects is not new. When different fraction schemes are used, it is valuable to be able to compare the relative acute and late effects of various fraction schemes. The concepts of nominal standard dose (NSD) and biologic-effective dose (BED) draw attention to the fact that the physical dose by itself is an inadequate representation of the effect that radiotherapy has on living tissues [20]. Radiation oncologists have understood that there is a difference between the acute tissues' response and late effects, but most regimens have been designed to balance acute-effect tissue response. The correlation between acute effects and late effects is imperfect. However, acute effect may parallel anti-tumor effect. Ideally therapy would produce no acute tissue damage, but it may be unrealistic to expect that there will be an absence of acute effects that still maintain anti-tumor effects. Today, we are better able to support patients through acutely toxic events. 
Standard or conventional dose radiotherapy has presently been established at 60 Gy in 2-Gy fractions. However, precise control rates in the chest and the exact dose limiting toxicity to each of the organs irradiated remain elusive with modern imaging and planning systems, some will attempt to re-establish partial lung tolerances for radiotherapy in standard fraction schemes by using 3-D treatment planning techniques. However, with standard fractionation treatment already lasts for 6 weeks. The prospect of accelerated repopulation of cancer cells during these protracted schedules has led some to explore accelerated fraction schemes, i.e. methods of giving intensified dose in shorter time. Hyperfractionation, slightly higher physical doses in the same overall time was standard fractionation, is very good for tumors with no radiobiologic shoulders, but hyperfractionation does not address the issue of accelerated repopulation of tumor cells. Accelerated fraction schemes have been used to treat small cell lung cancer, however, some may want to test a hyperfractionated scheme, particularly if normal tissue late effects to lung and spinal cord of the accelerated schedules seem excessive [19]. However, the total dose and duration of therapy may need to be explored. Furthermore, most view the late effects that have been achieved with these accelerated schedules to be fairly well-tolerated. Table 2 compares the advantages of accelerated versus hyperfractionated schemes. In general, there has been a movement away from the use of hypofractionation, except for palliation.

\section{A clinical example}

In 1984, at the University of Pennsylvania, a trial started comparing twice-a-day radiotherapy used concurrently with Platinum Etoposide to chemotherapy (cisplatin $60 \mathrm{mg} / \mathrm{m}^{2}$ on Day 1, Etoposide $120 \mathrm{mg} / \mathrm{m}^{2}$ Days 4,6 and 8 of each cycle). Two intense cycles of that chemotherapy were used; the radiotherapy overlapped with the first cycle [1]. There were no weekend treatments. Radiotherapy interruptions did not occur. Despite observed Cycle 1 toxicity, Cycle 2 chemotherapy was given in full doses on Day 22, i.e. two full courses without delays. Subsequently, six alternating cycles of platinum etoposide (PE) and cyclophosphamide, doxorubicin (Adriamycin), vincristine were alternated). A completion of approximately 6 months of therapy, prophylactic cranial irradiation (PCI) was applied to all surviving, completely responding patients.

\section{Table 2}

Hyperfractionation

Accelerated fractions

Excellent for sensitive tumors without shoulders.

Respects delicate surrounding late normal tissues (i.e. lung, spinal cord)

Higher physical dose

Time relatively unchanged from standard therapy
Sensitivity relatively less important

Attends to accelerated 'repopulations'

Lower physical dose increased biologic dose?

Decreased overall time

Relatively increased risk of acute toxicity 
The rationale for using the twice-daily radiotherapy was that the small fractions cause relatively less damage to surrounding normal tissues with a radiobiologic shoulder, such as lung and spinal cord, and even relatively acute-responding tissues, such as esophagus. On the other hand, small cell lung cancers without a shoulder were exponentially killed; and thus, did not have the opportunity to repair the damage that these relatively small doses per fraction provided. Also, since small cell lung cancer has the most prolific growth rate of any solid tumor [14], the potential for tumor growth between fractions of radiotherapy made allowed for passage from the relatively resistant S-Phase of the cell cycle to the more sensitive G2-M or M-Phase which is more radiosensitive.

Table 3 displays the PE Trials: five twice-a-day radiotherapy studies and two once-a-day radiotherapy studies. There appear to be improved outcomes between these trials and older cyclophosphamide-based trials. The 2-year survival is not dramatic between the daily and b.i.d. schedules. A number of factors differ between the trials including: PE-dose schedule; timing of radiotherapy; selection criteria; and duration of follow-up. The data presented show some differences in local control. However, the majority of failures remain systematic failures that lead to the largest cause of patient death. In order to clarify some of these issues, presently, active on-going trial continues in the ECOG, RTOG and SWOG cooperative groups that compares 45 Gy given in 3 weeks by a b.i.d. accelerated schedule versus 45 Gy given in 5 weeks in a standard schedule employing 1.8-Gy daily fractions. Early endpoints for toxicity, response and median survival appear similar; however, the longer term endpoints of 1,2,3 and 5 year disease free and overall survival, as well as local control, need more follow up and patient numbers to better evaluate the merit or risks of altered fraction schemes.

Table 4 displays four ways to deliver 45 Gy to the chest, as delivered in four reported trials. The Nominal Standard Dose methods have been used to compare

Table 3

Combined thoracic radiotherapy and platinum etoposide trials for limited small cell lung cancer

\begin{tabular}{|c|c|c|c|c|c|}
\hline B.I.D. studies & $\begin{array}{l}\text { No. of } \\
\text { Patients }\end{array}$ & Dose (Gy) & Time & 2-year survival & Local control \\
\hline
\end{tabular}

\begin{tabular}{|c|c|c|c|c|c|}
\hline Penn [18] & 32 & 45 Concurrent & 3 weeks & $48 \%$ & $84 \%^{\mathrm{a}}$ \\
\hline ECOG-1 [19] & 41 & 45 Concurrent & 3 weeks & $36 \%$ & - \\
\hline NCI-US [8] & 35 & 45 Concurrent & 3 weeks & $65 \%$ & - \\
\hline Mayo [7] & 36 & 48 Split course & 6 weeks & $40 \%$ & $97 \%$ \\
\hline ECOG-2 [9] & 41 & 45 Alternating & 8 weeks & $40 \%$ & - \\
\hline \multicolumn{6}{|l|}{ OD Studies } \\
\hline SWOG [13] & 154 & 45 Concurrent & 5 weeks & $42 \%$ & - \\
\hline Memorial [16] & 35 & 45 Sequential & 4-5 weeks & $46 \%$ & $74 \%$ \\
\hline
\end{tabular}

${ }^{a}$ All 4 variant histologies failed locally, only one $(3 \%)$ pure small cell failed locally.

BID, twice daily; OD, once daily; ECOG, Eastern Cooperative Oncology Group; SWOG, Southwest Coopcrative Oncology Group; NCI-U, National Cancer Institute United States; and Penn, University of Pennsylvania. 
Table 4

Relative scales to compare $45 \mathrm{~Gy}$ delivered in varied fraction sizes and times

\begin{tabular}{lllllr}
\hline Inst. & Fraction No. & Fraction size (Gy) & Time (D) & RET & NRET \\
\hline Memorial & 18 & 2.5 & 30 & 1546 & 1029 \\
SWOG & 25 & 1.8 & 33 & 1415 & 885 \\
PENN, ECOG-I & 30 & $1.5^{\mathrm{a}}$ & 19 & 1438 & 844 \\
ECOG II & 30 & $1.5^{\mathrm{a}}$ & 54 & 1282 & 793 \\
\hline
\end{tabular}

${ }^{a}$ Twice daily.

relative effects, the RET provides a relative scale for acute effects, and the Neuret provides a relative scale for late effects. These scales may have intrinsic flaws making their absolute values questionable, but they provide a reasonable mathematic model offering relative values for comparison of different fractionation time schedules. Most believed the $45 \mathrm{~Gy}$ given in b.i.d. fashion would be more acutely toxic than the same dose administered once each day in 18-Gy fractions - the two schemes used in the Intergroup Trial in the United States. The calculations predict very little differences between the two schedules, at least due to the radiotherapy factors (see SWOG vs. Penn or ECOG I, Table 4). Differences may also be influenced by the type of and timing with chemotherapy, as well as patient and tumor factors. Only trials will determine if 'breaks', time protraction of therapy, provide safer therapy or results in better, worse or similar tumor controls.

Prophylactic cranial irradiation (PCI) remains a contentious issue. If it is to be used, the relative effect of fractionation may be important. Even trials that have applied doses between 2000 and 3000 rad in 1-3 weeks report brain failures of approximately $20 \%$. Doses using larger dose per fraction are integrated with chemotherapy in ways that possibly interfere with blood/brain barrier protection have resulted in late imaging abnormalities and late neurologic toxicities $[6,11,17]$. There is relatively little information about using hyperfractionated schemes in prophylactic cranial irradiation. Since hyperfractionation is the best strategy to avoid unfavorable late effects, this may be a useful strategy. However, it would tax the patients to travel more frequently and undoubtedly is more expensive. Threegray or greater fractions are probably unwise, even when delayed to complexion of systemic therapy.

Perturbations in fractionation, overall time of therapy and integration of chemotherapy with local modality affords a rich rein to pursue in clinical research. There are rationales to increase effectiveness and decrease toxicity. Both are worthy of further investigation.

\section{References}

1 Arriagada R, Le Chevalier T, Ruffle $P$ et al. Alternating radiotherapy and chemotherapy in 173 consecutive patients with limited small cell lung carcinoma. Int J Radiat Oncol Biol Phys 1990; 19: 1135-8.

2 Bleehen NM, Bunn PA, Cox JD et al. Role of radiation therapy in small cell anaplastic carcinoma of the lung. Cancer Treat Rep 1983; 67: 11. 
3 Choi NC, Carey RC. Locoregional failure rate in relation with radiation dose in combined modality approach of multi-agent chemotherapy and radiotherapy for limited small cell lung carcinoma. Int J Radiat Oncol Biol Phys 1987; 13(s): 188-9.

4 Emami B, Lyman J, Brown A et al. Tolerance of normal tissue to thoracic irradiation. Int J Radiat Oncol Biol Phys 1991; 21: 109-122.

5 Emami B, Purdy JA, Mandis T et al. Three-dimensional treatment planning for lung cancer. Int J Radiat Oncol Biol Phys 1991; 21: 217-27.

6 Fleck JF, Einhorn LH, Lauer RC, Schuiz SM, Miller ME. Is prophylactic cranial irradiation indicated in small cell lung cancer? J Clin Oncol 1990; 8: 209 14.

7 Frytak S, Shaw E, Eagan R et al. Accelerated hyperfractionated split-course thoracic radiotherapy (AHSCTRT) and infusion cisplatin based chemotherapy (CT) for small cell lung cancer. Lung Cancer 1991; 7(S): 157 (Abstract).

8 Johnson BE, Salem C, Nesbitt J et al. Limited (LTD) stage small cell lung cancer (SCLC) treated with concurrent bid chest radiotherapy (RT) and etoposide cisplatin (VP/PT) followed by chemotherapy (CT) selected by in vitro drug sensitivity testing (DST). Lung Cancer 1991; 7(S): 152 (Abstract).

9 Johnson DH, Turrisi AT, Chang AY, Wagner H, Blum R. The eastern Cooperative Oncology Group. Alternating chemotherapy (CT) and Thoracic Radiotherapy (TRT) in limited small cell lung cancer. Lung Cancer 1991; 7(S): 155. (Abstract).

10 Lelieveld P, Scoles MA, Brown JM, Phil D, Kallman RF. The effect of treatment in fractionated schedules with the combination of X-irradiation and six cytotoxic drugs on the RIF-1 tumor and normal mouse skin. Int J Radiat Oncol Bio Phys 1985; 11: 111-21.

11 Lishner M, Feld R, Payne DG et al. Late complications after prophylactic cranial irradiation in patients with small-cell lung cancer: the Toronto expcrience. J Clin Oncol 1990; 8: 21521.

12 Looney WB, Hopkins HA. Alternation of chemotherapy and radiotherapy in cancer management. III. Results in experimental solid tumor systems and their relationship to clinical studies. Cancer Treat Rep 1986; 70: 141-62.

13 McCracken JD, Janaki LM, Crowley JJ et al. Concurrent chemotherapy/radiotherapy for limited small-cell lung carcinoma: a Southwest Oncology Group study. J Clin Oncol 1990; 8: 892.

14 Muggia F, Krezoski SK, Hansen H. All kinetic studies in patients with small cell carcinoma of the lung. Cancer 1974; 34: 1683-90.

15 Papac RJ, Son T, Biers R et al. Improved local control of thoracic disease in small cell lung cancer with higher dose thoracic irradiation and cyclic chemotherapy. Int J Radiat Oncol Biol Phys 1987; 13: $993-8$.

16 Shank B, Scher H, Hilaris RS et al. Increased survival with high-dose multifield and intensive chemotherapy in limited small cell carcinoma of the lung. Cancer 1985; 56: 2771.

17 Turrisi AT. Brain irradiation and systemic chemotherapy for small-cell lung cancer: dangerous liaisons? J Clin Oncol 1990; 8: 196-9.

18 Turrisi AT, Glover DJ. Thoracic radiotherapy variables: Influence on local control in small cell lung cancer limited disease. Int J Radiat Oncol Biol Phys 1990; 19: 1473-9.

19 Turrisi AT, Wagner H, Glover B et al. The Eastern Cooperative Oncology Group: Limited small cell lung cancer (LSCLC): Concurrent BID thoracic radiotherapy (TR) with platinum-etoposide (PE): An ECOG study. Proceedings of ASCO 1990; 9: 230 (Abstract 5).

20 Yaes RJ, Patel P, Maruyama T. On using the linear-quadratic model in daily clinical practice. Int J Radiat Oncol Biol Phys 1991; 20: 1353-8. 